\title{
Radiographic Evaluation of Dental Implants in the Periodontal Clinic Rumah Sakit Khusus Gigi dan Mulut (RSKGM) by the Faculty of Dentistry, Universitas Indonesia from 2009 to 2014
}

\author{
Stevany Grafiyanti ${ }^{1}$, Yuniarti Soeroso ${ }^{2}$, Hari Sunarto ${ }^{2}$ \\ ${ }^{1}$ Periodontics Residency Program, Faculty of Dentistry, University of Indonesia, Jakarta 10430, \\ Indonesia \\ ${ }^{2}$ Department of Periodontology, Faculty of Dentistry, University of Indonesia, Jakarta 10430, \\ Indonesia \\ *Email: yuniarti_22@yahoo.co.id
}

\begin{abstract}
Dental implant treatment is an alternative to tooth replacement with high success and survival rates. Evaluation of implant treatment is useful for predicting a treatment protocol that can determine the best surgical and implant systems. The purpose of this study was to evaluate implant treatments by analyzing the condition of the bones around dental implants using radiography and to determine the correlation of dental implant success with associated factors. Patients were recruited from the Periodontal Clinic Rumah Sakit Khusus Gigi dan Mulut (RSKGM), Faculty of Dentistry, Universitas Indonesia and screened for specific inclusion and exclusion criteria. A total of 22 ITI® Dental Implant Systems, which were placed from 2009 to 2014, were assessed. Radiographic evaluations were carried out using a Belmont Belray 096 standardized periapical radiographic dental x-ray unit and converted into digital images with a Digora ${ }^{\circledR}$ PCT digital imaging plate. Marginal bone loss was analyzed using Digora ${ }^{\circledR}$ for Windows software. The data were analyzed using IBM SPSS version 22. The mean marginal bone loss was $2.6 \pm 0.62 \mathrm{~mm}$ on the mesial aspect and $2.7 \pm 0.73$ $\mathrm{mm}$ on the distal aspect, and overall bone loss was $2.66 \pm 0.66 \mathrm{~mm}$. There were no statistically significant correlations between marginal bone loss and the location of the implant (maxilla or mandible), anteroposterior site, oral hygiene, or follow-up time. The dental implants used in the Periodontal Clinic RSKGM, Faculty of Dentistry, Universitas Indonesia demonstrated satisfactory results after 8 years.
\end{abstract}

Keywords: dental implant, radiographic evaluation, marginal bone loss, success criteria

\section{Introduction}

Clinically, replacing lost teeth with osseointegrated implants represents the most revolutionary advancement in restorative dentistry [1]. The procedure has proven to be a successful treatment modality, providing patients with a near natural replacement. Dental implants have become the treatment of choice when missing teeth require replacement $[1,2]$. A systematic review conducted by Moraschini et al. (2015) 
revealed a mean survival rate of $94.6 \%(\mathrm{SD}=5.97 \%)$ for a total of 7711 implants in 23 studies, with a follow-up period of up to 20 years (mean follow-up of 13.4 years) [3].

The term "implant success" may be used to describe the ideal clinical conditions. Implant success should include a time period of at least 12 months for implants serving as prosthetic abutments [4]. The term "implant survival" is used to describe dental implants that remained in place, i.e., were not removed for any reason. Commonly accepted criteria for the assessment of implant success were proposed by Albrektsson and colleagues [5]. Originally, a mean crestal bone loss of $\geqslant 1.5 \mathrm{~mm}$ during the first year after loading and $\geqslant 0.2 \mathrm{~mm} /$ year and no peri-implant radiolucency thereafter were proposed as the criteria for success $[5,6]$. The criteria introduced by Ross et al. (1997) was the amount of marginal bone loss around implants during the first year and during the entire 5-year observation time, classified into three categories: $\mathrm{I}=$ not more than $1.8 \mathrm{~mm}$, II = more than $1.8 \mathrm{~mm}$, and IIB $=$ more than $1.8 \mathrm{~mm}$ but less than $0.2 \mathrm{~mm}$ of resorption during the last year. The classification represented the worst case of mesial and/or distal bone height measurements [7].

Dental implants may fail for a number of reasons $[8,9]$. Factors thought to influence changes in marginal bone height after implant placement include delayed versus immediate implant placement, staging, timing of implant loading, requirement of bone graft at the implant site, presence of infection, medical conditions that compromise wound healing, smoking, status of oral hygiene, location of implant placement, and size of the implant. Other mechanical factors such as periosteum elevation during surgery, overheating of instruments resulting in osteonecrosis, occlusal trauma, the cantilever effect, and physiologic bone remodeling caused by inflammatory processes and plaque accumulation have also been suggested [10].

Radiographic assessments, along with annual follow-up examinations of patients treated with dental implants, have an important role [9] Evaluation of implant treatment is useful for assessing durability and long-term treatment success. It is also useful for determining the factors that influence the success of therapy and for identifying specific problems. Clinicians need long-term scientific evidence to determine the predictability of a treatment so that the best treatment protocol can be selected. The purpose of the present study was to evaluate peri-implant conditions radiographically and to determine their correlation with risk factors.

\section{Method}

Patients were selected according to the inclusion and exclusion criteria. The criteria for study inclusion were patients with an implant treatment carried out between 2009 and 2014 at the Periodontal Clinic Rumah Sakit Khusus Gigi dan Mulut (RSKGM), Universitas Indonesia and patients who could be contacted for followup. Patients were included in the study regardless of smoking status. Subjects with aggressive periodontitis, necrotizing ulcerative gingivitis (NUG), necrotizing ulcerative periodontitis (NUP), and diabetes mellitus were excluded from the study.

The study was comprised of 22 ITI ${ }^{\circledR}$ Dental Implant Systems (Institute Straumann AG, Waldenburg, Switzerland) placed in nine patients. Five patients (45.4\%) were female and six (54.5\%) were male. The median age was 53 years, ranging 
from 24 to 59 years. Seven implants were placed posteriorly $(68.2 \%)$ and 15 anteriorly (31.8\%). Eleven implants were maxillary (50\%) and $11(50 \%)$ mandibular.

All radiographs were taken at the Department of Radiology, Universitas Indonesia by an experienced radiologist using a Belmont Belray 096 dental x-ray unit (Belmont, Takara Belmont Corp. Japan) with an exposure time of 0.18 seconds. The radiograph was taken using the long cone paralleling technique and was converted into digital images with an imaging plate, Digora ${ }^{\circledR}$ PCT (SOREDEX, PaloDEx Group Oy, Tuusula, Finland). An effort was made to attain clearly visible threads. No further attempts were made to standardize measurements. No bite block or special aiming devices were used.

Radiographic parameters were evaluated using Digora ${ }^{\circledR}$ for Windows software. Landmarks were identified by two interpreters who reached a consensus $[11,12]$ Linear measurements between landmarks were taken medially and distally from the implant shoulder to the first visible apical bone-to-implant contact (BIC), assuming that baseline bones were at the implant shoulder. The distance was measured in millimeters.

The technical measurement of error (TEM) of radiographic measurements was calculated by comparing the values of the first and second interpreter's radiographic readings using the Dahlberg formula (Fig 1). All the recorded radiographic parameters were entered into the standard perform drawn for this study and subjected to statistical analysis.

$$
d=\sqrt{\frac{\sum_{\mathrm{i}=1}^{\mathrm{n}}\left(\mathrm{X}_{1 \mathrm{i}}-\mathrm{X}_{2 \mathrm{i}}\right)^{2}}{2 \mathrm{n}}}
$$

Fig. 1 Dahlberg formula used to calculate the technical measurement of error (TEM).

The data were grouped according to the implant's jaw location (maxilla or mandible), the anteroposterior site, and oral hygiene scores. Oral hygiene scores were determined using the Silness-Löe plaque index (poor, fair, good), correlations with mean bone loss calculated using Spearman's rank correlation coefficient, and follow-up times using Pearson's correlation coefficient. Statistical significance was set at $\mathrm{p}<0.05$ for all statistical tests.

\section{Results}

In this study, the survival rate of implants was $100 \%$. Forty-four radiographic implant readings, which consisted of mesial and distal aspects, were interpreted by the first and second interpreter. The mean difference between the first and the second interpreters' mesial aspect readings was $-0.4 \pm 0.81 \mathrm{~mm}$, with readings ranging from -3.26 to $0.66 \mathrm{~mm}$. The mean difference in marginal bone loss in the distal aspect was $0.44 \pm 0.77 \mathrm{~mm}$, ranging from -3.58 to $0.49 \mathrm{~mm}$. The relative technical error of measurement (TEM) for the mesial and distal aspects was 0.62 and 0.61 , 
respectively. For descriptions of observed bone loss, only the results obtained by the first interpreter were used.

The mean marginal bone loss was $2.6 \pm 0.62 \mathrm{~mm}$ in implants evaluated in the mesial aspect and $2.7 \pm 0.73 \mathrm{~mm}$ in those evaluated in the distal aspect. Thus, the total mean bone loss was $2.66 \pm 0.66 \mathrm{~mm}$ (0le 1). The largest amount of bone loss was $4.04 \mathrm{~mm}$ for implants evaluated in the mesial aspect and $3.96 \mathrm{~mm}$ for those evaluated in the distal aspect (Graph 2). Variables such as location of the implant (maxilla, mandibula, anteroposterior), oral hygiene, and follow-up time had no significant correlation with peri-implant marginal bone loss.

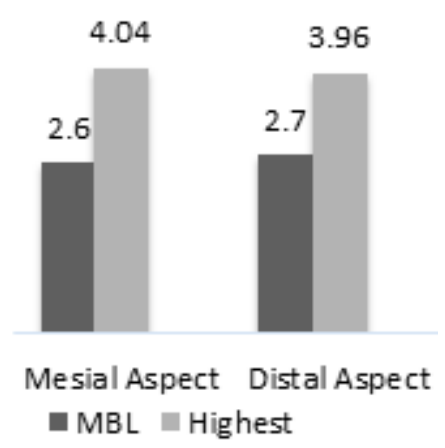

Graph. 2 Comparison between mean bone loss (MBL) and highest bone loss

\section{Discussion}

The present study involved a retrospective analysis of peri-implant conditions in patients with dental implants. A cross-sectional, observational design was chosen. The study was descriptive, and its purpose was to investigate associations between risk factors and outcomes of interest. The study design was cross-sectional and, as such, gives no indication of sequences of events or whether exposure occurred before, after, or during the onset of disease outcomes. This study did not systematically evaluate initial conditions or the standardization of clinical procedures. Marginal bone loss is typically measured during annual implant follow-ups $[13,14]$ Marginal bone loss that occurred the first year after prosthetic loading and every year thereafter were calculated. In this study, the follow-up time was the time from implantation until the patient came to RSKGM for follow-up. It was difficult to determine the time of implantation due to a lack of recorded data; therefore, annual bone loss could not be determined.

The subjects of this study were recruited from the Periodontal Clinic Rumah Sakit Khusus Gigi dan Mulut, Universitas Indonesia. As expected, in some cases, analysis of clinical histories was complicated by incomplete information and suboptimal recording of data, similar to other studies where there was no standardization of data recording [15]. Only nine patients could be contacted to come into the clinic for follow-up. The mean patient age was 47.2 years (range: $24-59$ years). This range could suggest selection bias. However, most individuals were in their $40 \mathrm{~s}$. 
The precision of the peri-implant bone level readings, determined by the first and second interpreter, reflected a learning curve in defining reference points. Methodological limitations may result in false diagnoses when assessing small peri-implant bone height changes. False diagnoses may be related to measurement errors and/or variation in projections.

When repeated measurements are taken of the same object, the value obtained will vary. This phenomenon is referred to as the technical error of measurement (TEM). Technical errors of measurement may be used to determine inter-observer errors that occur when two workers independently measure the same things. In this study, this measurement was calculated using the Dahlberg formula.

In this study, the mean follow-up was 3.2 years, the mean mesial marginal bone loss was $2.6 \pm 0.2 \mathrm{~mm}$, and the mean distal bone loss was $2.7 \pm 0.73 \mathrm{~mm}$. This result is higher than the mean marginal bone loss of $1.8 \mathrm{~mm}$ found by Roos et al. (1997) after 5 years of follow-up. However, there was no clinical impact of bone loss since the majority of implants had no clinical inflammation. Factors that contribute to marginal bone loss are poor oral hygiene, occlusal overload, immediate implant placement, staging, timing of implant loading, requirement of a bone graft at the implant site, presence of infection, medical conditions, smoking status, location of implant placement, inter-implant distance, implant-tooth distance, and design and size of the implants [10]. In this study only the location (maxilla or mandible), anteroposterior site, oral hygiene, and follow-up time were assessed. Statistical analyses showed a weak correlation between marginal bone loss and oral hygiene $(\mathrm{r}=0.16)$, follow-up time $(\mathrm{r}=0.06)$, location in the jaw $(\mathrm{r}=-0.02)$ and anteroposterior site $(\mathrm{r}=0.16)$. These results are in agreement with those of previous reports.16 Fifty years after the advent of osseointegration, peri-implantitis is a minor problem, affecting only $1 \%-2 \%$ of modern implants in 10 years [17]. This statistic is supported by our study, suggesting that oral hygiene has a statistically insignificant correlation with marginal bone loss.

\section{Conclusion}

Radiographic evaluation of ITI ${ }^{\circledR D}$ Dental Implants in the Periodontal Clinic Rumah Sakit Khusus Gigi dan Mulut, Universitas Indonesia demonstrated a satisfactory result after 8 years.

\section{References}

1. Rajpal J, Gupta KK, Tandon P, Srivastava A, Chandra C. Assessment of hard and soft tissue changes around Implants: A clinico-radiographic in vivo study. 2014;4(2):168-72.

2. Sanjna N, Ramakrishna R. Clinical assessment of primary stability of endosseous implants placed in the incisor region, using resonance frequency analysis methodology: An in vivo study. Indian J Dent Res. 2007;18(4):168-73.

3. Moraschini V, Poubel LAC, Ferreira VF, Barboza ESP. Evaluation of survival and success rates of dental implants reported in longitudinal studies with a follow-up period of at least 10 years : a systematic review. Int J Oral Maxillofac Surg. 2015;(October 2014):377-88.

4. Misch CE, Perel ML, Wang H, et al. Implant Success, Survival, and Failure: Implantologists (ICOI) Pisa. 2008:5-15.

5. Albrektsson T, Eriksson AR, Zarb G, Worthington P. the long term efficacy of currently 
used dental implant a review and proposed criteria of success. Int J Oral Maxillofac Implants. 1986;1(1):11-25.

6. Gallucci GO. Success Criteria in Implant Dentistry: A Systematic Review. 2012:242-9.

7. Roos J, Sennerby L, Lekholm U, Jemt T, Grondahl K, Albrektsson T. A Qualitative and Quantitative Method for Evaluating Implant Succes: A 5-year Rertrospective Analysis of the Brånemark Implant. Int J Makxillofac Implant. 1997;12(4):504-514.

8. Babbush CA, Hahn JA, Krauser JT, Rosenlicht JL. Dental Implants The Art and Science. 2nd ed. Maryland Height: Elsevier Inc.; 2011.

9. Misch CE. Cotemporary Implant Dentistry. 3rd ed. St. Louis Missouri: Elsevier Inc.; 2008.

10. Kim S, Dodson T, Do D, Wadhwa G, Chuang S. Factors associated with crestal bone loss following dental implant placement. J Oral Maxillofac Surg. 2011;69(1):e-56. doi:10.1563/AA1D-JOI-D-12-00193.

11. Blanes RJ, Bernard JP, Blanes ZM, Belser UC. A 10-year prospective study of ITI dental implants placed in the posterior region: Clinical and radiographic results. Clin Oral. 2007;18:699-706. doi:10.1111/j.1600-0501.2006.01306.x.

12. Bragger U, Hafeli U, Huber B, Hammerle CHF, Lang NP. evaluation of crestal bone level.pdf. Clin Oral Implants Res. 1998;9:218-224.

13. Park W, Park MSY. A 10-Year Retrospective Radiographic Study of Implantium Dental Implants. 2015;35(1):49-55. doi:10.11607/prd.prd.2289.

14. Geraets W, Zhang L, Wismeijer D. Annual bone loss and success rates of dental implants based on radiographic measurements. Dentomaxillofacial Radiol. 2014;43:1-7. doi:10.1259/dmfr.20140007.

15. Abreu MH, Bianchini MA, Magini RS, Rösing CK. Clinical and Radiographic Evaluation of Periodontal and Peri-Implant Conditions in Patients with Implant-Supported Prothesis. 2007;20:87-95.

16. Nemli SK, Gungor MB, Aydin C, Yilmaz H, Bal BT, Arici YK. Clinical and radiographic evaluation of new dental implant system: Results of a 3-year prospective study. J Dent Sci. 2016;11(August 2013):29-34.

17. Albrektsson T, Buser D, Sennerby L. Crestal Bone Loss and Oral Implants. Clin Implant Dent Relat Res. 2012;14(6):783-792. doi:10.1111/cid.12013. 